\title{
La letteratura d'impegno: la nascita e lo sviluppo
}

\author{
Ahmed Shehata Ibrahim \\ Assistente nel dipartimento italiano
}

Innanzitutto dobbiamo affermare che l'impegno letterario non è una corrente letteraria tutta moderna, ma è una corrente nata con la nscita della letteratura. Giulio Ferroni, in un'intervista, rispondendo alla domanda: Che rapporto c'è fra letteratura italiana e impegno dice:

Per parlare di impegno nella letteratura italiana bisogna risalire a Dante, il cui impegno etico e politico attraversa tutta la Commedia e arriva fino alla cantica più lontana dal mondo, il Paradiso. Ma dopo Dante c'è una svolta: Petrarca impone il modello dell'autosufficienza della letteratura, modello che perdura nei secoli, fino a Foscolo, con cui inizia il tramonto del "letterato", e che sarà distrutto dal Risorgimento, quando gli scrittori si sentiranno in dovere di partecipare alla lotta per l'unità d'Italia. Fino ad arrivare al Novecento, che inizia con avanguardia e politicizzazione, quindi con una diretta immersione nell'onda della storia, e al '68, con un ulteriore rilievo dato alle istanze della politica ${ }^{1}$.

All'inizio del mio lavoro mi sono subito interrogato sul significato che la parola "impegno" avrebbe potuto assumere in riferimento ai vari autori.

Con il termine "intellettuale" non voglio intendere solo la figura del filosofo: il filosofo che pensa, che formula idee, ma sono più propenso a vedere l'intellettuale in un ambito più ampio: L'intellettuale può essere definito come un uomo di cultura, in grado di spiegare in modo totalmente letterario le psiche e i sentimenti delle persone. Una personalità che ha la possibilità di aiutare la società ad arrivare alla salvezza. Esattamente come dice Carlo Cassola: " la salvezza - avverte Cassola - non potrà venire che da "un piccolo gruppo di uomini di cultura" capace di influenzare "vaste masse". 2

L'intellettuale è una personalità che ha a che fare col pensiero astratto, con valori alti e profondi, con storie e parole che scandagliano le più intime profondità delle emozioni umane, della psiche, dei sentimenti; ma anche della società e della politica passata e presente: 
Io so perché sono un intellettuale, uno scrittore, che cerca di seguire tutto ciò che succede, di conoscere tutto ciò che se ne scrive, di immaginare tutto ciò che non si sa o che si tace» ${ }^{3}$.

La società, l'ambiente circondante influenzano la figura dell'intellettuale. Non si si può parlare di intellettuale definitivamente impegnato senza vedere il contesto storico in cui vive. Ermanno $\mathrm{Rea}^{4}$ dice:

Non si può parlare di intellettuale impegnato isolandolo dalla sua communitas. Quanto più la sua comunità di elezione sarà costituita da cittadini responsabili ed attenti al bene collettivo, tanto più l'intellettuale sarà in grado di contribuire mettendo a disposizione degli altri le sue competenze. ${ }^{5}$.

Il Novecento è stato senza dubbio un'epoca di crisi, di complessità, di fatti storici grandiosi e drammatici, come i totalitarismi e i due conflitti mondiali; questi eventi, oltre ad avere molte influenze brutte e causato un gran numero di morti e disillusioni di ogni genere, hanno anche notevolmente influito sulla mentalità e sui valori ideologici delle popolazioni.

Ho voluto, con il mio percorso, andare ad esplorare come l'intellettuale si è posto nei confronti di tale scenario e come è nata la letteratura d'impegno.

L'arco di tempo fu molto vasto, le situazioni sono state numerose ed è per questo che ho deciso di dare luce sul periodo che andava dall'inizio della prima guerra mondiale al periodo della secondo dopoguerra.

Dopo la conclusione del conflitto mondiale e con alle spalle l'esperienza recentissima e sofferta della Resistenza che aveva visto la partecipazione di gruppi politicamente eterogenei alla lotta al nemico comune, si instaurò, come sappiamo, in Italia un clima di solidarietà "emotiva" che visse convergere una larga fascia di intellettuali, all'insegna di un ancora fresco antifascismo militante e nella prospettiva di uno spontaneo ma a volte generico impegno politico, nelle non ancora ben definite direttive culturali del partito comunista. In un'Italia ancora sconvolta dalle ripercussioni della guerra e da anni di dittatura fascista, era infatti prevedibile che l'intellettuale si assumesse il compito di rinnovare e di ricostruire la società tramite una rivalutazione della propria funzione culturale ed 
un'impostazione "a sinistra" del proprio programma politico. I1 PCI, dunque, in quanto esponente di spicco della scena politica italiana, costituisce il denominatore comune nel quale si identificano tutti quegli uomini di cultura propensi, al di là delle loro caratterizzazioni strettamente ideologiche e in quel clima di solidarietà ereditato dalla lotta partigiana, ad indirizzare le proprie forze verso un concreto impegno civile e sociale.

La situazione della società italiana che si creò in conseguenza della Resistenza e della ripresa democratica aveva dirette ripercussioni sulla vita letteraria ed artistica: iniziava un nuovo corso che si è soliti indicare come "l'età del Neorealismo".

In sintesi con il Neorealismo si intese rappresentare la realtà italiana con tutto quello che di nuovo presentava. Il Neorealismo che abbracciava il decennio che andava dal 1945 al 1955, non era né una scuola né un gruppo di scrittori operanti secondo un programma e una poetica comuni, ma si trattava di uno stato d'animo collettivo, di un'esigenza, sincera anche se spesso confusa, di impegno.

Condivido il giudizio di un critico, Manacorda, il quale considera il Neorealismo "come una disposizione più pratica, eticopolitica, che non estetica, più di una unzione della volontà che non della fantasia o dell'intelletto"6.

In questi anni assistiamo infatti a un dibattito molto intenso sulle scelte degli intellettuali contro i totalitarismi a favore della libertà. Nacque così una cultura, dunque, "impegnata", ma autonoma nei confronti della politica, poiché la cultura "non può non svolgersi all'infuori da ogni legge di tattica e di strategia, sul piano diretto della storia"?

Quindi siamo davanti a un periodo molto sensibile nella storia della letteratura moderna, che aveva creato un intellettuale che faceva parte della propria società, cioè, l'intellettuale impegnato.

L'intellettuale di quel periodo fu uomo di cultura molto attivo, non isolato e rinchiuso nella sua torre d'avorio, un intellettuale progressista, la cui funzione "era, ovunque, di combattere per migliorare la società ed eliminarne i difetti", e rivoluzionario, 
proponendo però qualcosa di diverso dalle esigenze proposte dalla politica:

L'intellettuale "impegnato," dunque, soprattutto se contraddittoriamente polemico e poco accomodante dal punto di vista ideologico, visse problematicamente questa "involuzione" politica come militante iscritto al partito ma anche come scrittore e uomo di cultura che inizialmente aveva trovato nel mito della politica la giustificazione immediata al bisogno impellente di comunicazione con gli altri e che ora si ritrova a vivere una condizione di inquietudine generale e di alienazione profonda una volta svaniti quei valori e quelle sicurezze che erano stati il supporto ideologico ed "emotivo" del periodo resistenziale ${ }^{8}$.

L'intellettuale, il letterato o l'artista furono fortemente impegnati, portatori di un messaggio, engagé, per i quali l'opera contava solo in quanto recava un apporto pratico alla trasformazione degli uomini e alla storia. Attività culturale ed azione politica apparirono come elementi di un processo storico innovato nel quale il nuovo intellettuale svolgeva un ruolo importante .

Sempre Vittorini avrebbe detto che l'impegno dell'intellettuale non deve essere "suonare il piffero della rivoluzione", ma deve consistere piuttosto in un "engagement naturale che agisce in lui al di fuori della sua volontà. Gli viene dall'esperienza collettiva di cui egli è spontaneo portatore, e costituisce, segreto in lui stesso, l'elemento principale della sua attività"10.

Per tutto questo possiamo dire che l'intellettuale impegnato è quello che "non sta nella torre eburnea, avulso dal contesto, che non si compromette con le vicende storiche... "11.

La scelta dei temi fu caratterizzata da una vera e propria fame di realtà, di quella realtà che urgeva ed era sotto gli occhi di tutti: la guerra, la resistenza, la lotta per sbarcare il lunario giorno per giorno, le città devastate, le grandi masse che, diventate protagoniste di storia, impegnarono le loro lotte.

La letteratura impegnata del dopoguerra si sviluppò un vivace dibattito letterario caratterizzato in primo luogo dalla politicizzazione del letterato. Si può affermare che, la letteratura, come la cultura andavano risolutamente a Sinistra. Il Paese avvertò il bisogno di rivedere la propria storia; si avrebbero così nuove 
interpretazioni del Risorgimento, e successivamente, del brigantaggio e della questione Meridionale, poi delle lotte operaie, del ventennio fascista e della Resistenza:

Tra la fine della guerra e il maggio francese, nella letteratura italiana sfilarono diverse modalità di contestazione dello status quo politico e sociale: dalle impegnate denunce del Neorealismo ai romanzi dell'alienazione, che segnarono gli anni del boom; sino ad arrivare alle negazioni radicali propugnate dalla Neoavanguardia, con il Gruppo 63."12

I giovani scrittori, provati dall'esperienza della guerra, dalla prigionia, dalla Resistenza, avvisarono il bisogno di recuperare la realtà sociale. Questi argomenti diventarono così molto spesso, occasioni e temi narrativi affidati al romanzo che, proprio in questi anni, fu protagonista della scena. Inoltre, il rivolgersi del paese a una politica moderata e avara delle riforme di fondo di cui si avvertiva la necessità, orientò molti scrittori verso un'adesione al Partito comunista, che nacque, spesso, da un generico entusiasmo e da una volontà d'azione, piuttosto che di una vera accettazione del Marxismo, e diede presto origine a crisi e ripensamenti, già evidenti in Pavese e Vittorini.

Intellettuali come Pavese e Vittorini si iscrivono nel dopoguerra al PCI: Pavese si iscrissero al partito nel '45 e ne diventò attivista e quadro, esprimendo la sua partecipazione con interventi di carattere politico e sociale, e collaborò al quotidiano "l'Uunità", mentre Vittorini andò a Milano nel 1942 e prese parte alla Resistenza, Nel '45 scrisse "Uomini e no" e si iscrisse al partito comunista:

La questione complessa del rapporto tra cultura e politica e l'influenza che tale polemica esercitò all'interno del dibattito tra intellettuali e potere, interessò e coinvolse più da vicino soprattutto Elio Vittorini. I1 nome di Vittorini è quello che più si avvicina, infatti, anche se partendo da impostazioni culturali e letterarie diverse, alle stesse controverse questioni che interessarono la vicenda intellettuale di Pavese ${ }^{13}$.

In questo periodo la politica culturale del PCI propese inoltre verso una diffusione della dottrina marxiana. Nacquero dovunque centri di cultura dove si discusse di marxismo, di esistenzialismo, di nuovo impegno politico dei cattolici e di letteratura. Il comunismo rappresentava per gli intellettuali impegnati un punto di riferimento, ma in tanti tempi il comunismo fu punto di 
disaccordo tra gli scrittori impegnati stessi. Se da un lato, infatti, Pavese scrisse: "La storia anche italiana di questi ultimi anni dimostra a chi vuol vedere che gli intellettuali posson trovare nel comunismo il più efficace strumento per realizzare una concreta libertà intellettuale"'14. Dall'altro, lo scrittore siciliano affermò: Perciò è inutile che si parli di "via democratica" al comunismo, di "via democratica" al socialismo. A noi (agli uomini) interessa che il comunismo stesso e il socialismo stesso siano "democratici." Questo intendiamo dire dicendo che li pensiamo come '"vie."15.

Le riviste giocarono anche un ruolo molto importante in quel periodo. Fra le numerose riviste coinvolte in quest'opera di rinnovamento culturale, una delle più importanti era senza dubbio "IL POLITECNICO", diretta da Elio Vittorini, uscita dal 29 settembre 1945 come settimanale, e poi, dal 1 maggio 1946 al dicembre 1947, come mensile. Facendosi portavoce degli intellettuali usciti dalla Resistenza, si poneva come obiettivo la fondazione di una cultura in grado di creare un connubio tra le istanze scientifiche e quelle umanistiche, tra la letteratura e il pensiero scientifico; in pratica una cultura attiva nei confronti dell'uomo e della società, combattendo il disimpegno precedente. 


\section{Bibliografia}

1 Bianca Garavelli, Letteratura tra autosufficienza e impegno - Letture n. 654, febbraio 2009, http://www.biancagaravelli.it/articoli/letteratura-autosufficienza-impegno-letture.php

2 R. Luperini, in Carlo Cassola, Atti del Convegno-Firenze, Palazzo Medici-Riccardi, 3-4 novembre 1989, Pontassieve, Becocci Editore, (a cura di Giovanni Falaschi), p. 35.

${ }^{3}$ Pier Paolo Pasolini, il corriere della sera, 14 Novembre 1974, Cos'è questo golpe? Io so.

${ }^{4}$ Ermanno Rea è nato a Napoli nel 1927. Ha lavorato come giornalista, per numerose testatequotidiani e settimanali. Ha vissuto a Milano e a Roma. I suoi libri sono prevalentemente inchieste su casi personali (la militante comunista Francesca Spada, suicida, in Mistero napoletano, il docente di economia Federico Caffè, scomparso misteriosamente, in L'ultima lezione che permettono di raccontare la realtà circostante.

${ }^{5}$ Ermanno Rea, Micromega, l'intellettuale e l'impegno, 6/2013

${ }^{6}$ Giuliano Manacorda, Storia della letteratura italiana contemporanea, 1967, Editori Riuniti, Roma, p. 28.

${ }^{7}$ E. Vittorini, "Politica e cultura", in Il Politecnico, Milano, Lerici, 1960, p. 126

8 Vincenzo Binetti, Marginalità e appartenenza: la funzione dell'intellettuale tra sfera pubblica e privato nell'Italia del dopoguerra, Italica, Vol. 74, No. 3. (Autumn, 1997), pp. 365.

${ }^{9}$ E. Vittorini, "Politica e cultura. Lettera a Togliatti", in Il Politecnico, Milano, Lerici, 1960, p. 189

${ }^{10}$ E. Vittorini, Diario in pubblico, Milano, Bompiani, 1957, p. 306.

${ }^{11}$ Cfr., Pellegata Maria Beatrice, l'impegno degli intellettuali nel '900, Tesina Esame di Stato, 2001-2002, liceo scientifico " BRAMANTE" di MAGENTA.

12 M. Novelli, "La letteratura italiana tra impegno e contestazione (1944-1967)" in Il corriere della sera, 18/12/2007.

13 Vincenzo Binetti, Marginalità e appartenenza: la funzione dell'intellettuale tra sfera pubblica e

privato nell'Italia del dopoguerra, op. cit., . pp. 365- 366.

${ }^{14}$ Ivi., p. 367

${ }^{15}$ Ibidem. 